$\exists \int$ Journal of Logic \& Analysis 4:5 (2012) 1-6

ISSN 1759-9008

\title{
Generating the Pfaffian closure with total Pfaffian functions
}

\author{
GARETH JONES \\ PATRICK SPEISSEGGER
}

\begin{abstract}
Given an o-minimal expansion $\mathcal{R}$ of the real field, we show that the structure obtained from $\mathcal{R}$ by iterating the operation of adding all total Pfaffian functions over $\mathcal{R}$ defines the same sets as the Pfaffian closure of $\mathcal{R}$.
\end{abstract}

2010 Mathematics Subject Classification 14P10,03C64 (primary); 58A17 (secondary)

Keywords: o-minimal structure, Pfaffian function, Rolle leaf

There are various possibilities for adding Pfaffian objects to o-minimal expansions of the real field and preserving o-minimality. One example is the Pfaffian closure of an o-minimal expansion of the real field, which was shown to be o-minimal by the second author [7]. The purpose of this note is to present a somewhat simpler construction of the Pfaffian closure. Although not as simple as the description in terms of nested leaves obtained by Lion and the second author [5], our construction has the novelty of only using total Pfaffian functions and is reminiscent of the original Pfaffian expansion of the real field constructed by Wilkie [9].

In order to state our result, we need to introduce some terminology. Suppose that $\mathcal{R}$ is an o-minimal expansion of the real field, and that $U \subseteq \mathbb{R}^{n}$ is an $\mathcal{R}$-definable open subset of $\mathbb{R}^{n}$ for some $n \in \mathbb{N}$. We say that a $C^{1}$ function $f: U \rightarrow \mathbb{R}$ is Pfaffian over $\mathcal{R}$ if there exist $\mathcal{R}$-definable $C^{1}$ functions $P_{i}: U \times \mathbb{R} \rightarrow \mathbb{R}$, for $i=1, \ldots, n$ such that

$$
\frac{\partial f}{\partial x_{i}}(x)=P_{i}(x, f(x))
$$

for all $x \in U$.

Given $n, l \in \mathbb{N}$ such that $l \leq n$, we let $G_{n}^{l}$ be the Grassmannian of all linear subspaces of $\mathbb{R}^{n}$ of dimension $l$. This is an analytic manifold and is naturally definable in the real field (see [1,3.4.2]). We also set $G_{n}=\bigcup_{l=0}^{n} G_{n}^{l}$. Now fix an embedded $C^{1}$ submanifold $M$ of $\mathbb{R}^{n}$ and let $l \leq n$. A $C^{1}$ map $d: M \rightarrow G_{n}$ is said to be a distribution on $M$ if $d(x) \subseteq T_{x} M$ for all $x \in M$, where $T_{x} M$ is the tangent space of $M$ at $x$. A distribution $d$ is an l-distribution if $d(M) \subseteq G_{n}^{l}$. Given an $l$-distribution $d$ on $M$ and an immersed $C^{1}$ submanifold $V$ of $M$, we say that $V$ is an integral manifold of $d$ if $T_{x} V=d(x)$ for all 
$x \in V$. A maximal connected integral manifold is called leaf of the distribution. Now suppose that $d$ has codimension one. A leaf $L$ of $d$ is said to be a Rolle leaf of $d$ if it is a closed embedded submanifold of $M$ and is such that for all $C^{1}$ curves $\gamma:[0,1] \rightarrow M$ satisfying $\gamma(0), \gamma(1) \in L$, we have $\gamma^{\prime}(t) \in d(\gamma(t))$ for some $t \in[0,1]$. A Rolle leaf over $\mathcal{R}$ is a Rolle leaf of an $\mathcal{R}$-definable codimension one distribution defined on $\mathbb{R}^{n}$ for some $n \in \mathbb{N}$. For example, a result due to Khovanskii (see $[8,1.6])$ implies that if $f: \mathbb{R}^{n} \rightarrow \mathbb{R}$ is Pfaffian over $\mathcal{R}$, then the graph of $f$ is a Rolle leaf over $\mathcal{R}$.

We can now define the Pfaffian structures involved in our result. Given any o-minimal expansion of the real field $\mathcal{R}$, let $\mathcal{L}(\mathcal{R})$ be the collection of all Rolle leaves over $\mathcal{R}$. Now let $\mathcal{R}_{0}=\mathcal{R}$ and, for $i \geq 0$, let $\mathcal{R}_{i+1}$ be the expansion of $\mathcal{R}_{i}$ by all leaves in $\mathcal{L}\left(\mathcal{R}_{i}\right)$. Let $\mathcal{L}$ be the union of all the $\mathcal{L}\left(\mathcal{R}_{i}\right)$ and let $\mathcal{P}(\mathcal{R})$ be the expansion of $\mathcal{R}$ by all the leaves in $\mathcal{L}$. This structure is called the Pfaffian closure of $\mathcal{R}$. The second author showed that it is o-minimal [7].

Similarly, we let $\mathcal{L}^{\prime}(\mathcal{R})$ be the collection of all functions $f: \mathbb{R}^{n} \rightarrow \mathbb{R}$, for all $n \in \mathbb{N}$ that are Pfaffian over $\mathcal{R}$. We define $\mathcal{R}_{i}^{\prime}$ and then $\mathcal{P}^{\prime}(\mathcal{R})$ by mimicking the previous paragraph. The structure $\mathcal{P}^{\prime}(\mathcal{R})$ is a reduct of $\mathcal{P}(\mathcal{R})$ (by the example above) and it is the purpose of this note to show that they are in fact the same from the point of view of definability.

Theorem 1 A set $X \subseteq \mathbb{R}^{n}$ is definable in $\mathcal{P}(\mathcal{R})$ if and only if it is definable in $\mathcal{P}^{\prime}(\mathcal{R})$.

If $\mathcal{R}$ admits analytic cell decomposition, then so too does $\mathcal{P}^{\prime}(\mathcal{R})$ (see [8]) and it follows that in this case, the reduct of $\mathcal{P}^{\prime}(\mathcal{R})$ in which only analytic functions are added also defines the same sets as $\mathcal{P}(\mathcal{R})$.

Before proving the theorem, we first recall a result from Khovanksii theory that we will repeatedly use in the proof. This originates with Khovanskii's work on pfaffian functions (see [3]) and was adapted to the o-minimal setting by the second author [7] following work of Moussu and Roche [6] and Lion and Rolin [4]. In our proof we shall only ever need to work with a single distribution so we restrict ourselves to Khovanksii theory in this simple setting. We follow the presentation in [8]. Suppose that $d$ is a $C^{2}$ distribution on a $C^{2}$ manifold $M \subseteq \mathbb{R}^{n}$ and that $N \subseteq M$ is a $C^{2}$ submanifold of $M$. The pull-back of $d$ to $N$ is the distribution $d^{N}$ on $N$ defined by

$$
d^{N}(x)=T_{x} N \cap d(x) .
$$

We say that $N$ is compatible with $d$ if $d^{N}$ is an $l$-distribution on $N$, for some $l$. From now on, we use the word definable to mean $\mathcal{P}^{\prime}(\mathcal{R})$-definable. In particular, cell means $\mathcal{P}^{\prime}(\mathcal{R})$-definable cell. The result we need is as follows (see $[8,3.6]$ ). 
Fact 2 Let $d$ be a definable codimension one distribution on $M$ and suppose that $\mathcal{C}$ is a partition of $M$ into $C^{2}$ cells compatible with $d$. Then there is a $k \in \mathbb{N}$ such that whenever $C \in \mathcal{C}$ and $L$ is a Rolle leaf of $d$ the set $C \cap L$ is a union of at most $k$ Rolle leaves of $d^{C}$.

Given the definition of $\mathcal{P}^{\prime}(\mathcal{R})$, in order to prove the theorem it suffices to show that if $L$ is a Rolle leaf over $\mathcal{P}^{\prime}(\mathcal{R})$ then $L$ is definable. For the proof of this, we assume that the reader is familiar with o-minimality (as presented in [2]). First, an easy observation.

Lemma 3 Suppose that $C \subseteq \mathbb{R}^{n}$ is an open $C^{2}$ cell and that $f: C \rightarrow \mathbb{R}$ is Pfaffian over $\mathcal{P}^{\prime}(\mathcal{R})$. Then $f$ is definable.

The proof, using a definable diffeomorphism between $C$ and $\mathbb{R}^{n}$, is left to the reader.

Now suppose that $C \subseteq \mathbb{R}^{n}$ is a bounded open $C^{2}$ cell, and that $\alpha, \beta, \gamma, \delta: C \rightarrow \mathbb{R}$ are definable bounded $C^{2}$ functions such that

$$
\gamma(x)<\alpha(x)<\beta(x)<\delta(x)
$$

for all $x \in C$. Let $D=(\alpha, \beta)_{C}$ and $D^{\prime}=(\gamma, \delta)_{C}$ and suppose that $d^{\prime}$ is a definable integrable $n$-distribution on $D^{\prime}$ (for a discussion of integrability in this context, see [8, Section 1]). Suppose that we are given a Rolle leaf $L^{\prime}$ of $d^{\prime}$. Assume that both the graph of $\alpha$ and the graph of $\beta$ are compatible with $d^{\prime}$ and let $d^{\alpha}$ and $d^{\beta}$ be the pullbacks of $d^{\prime}$ to the graphs of $\alpha$ and $\beta$ respectively. Let $d$ be the restriction of $d^{\prime}$ to $C$. By Fact $2, L^{\prime} \cap D, L^{\prime} \cap$ graph $\alpha$ and $L^{\prime} \cap \operatorname{graph} \beta$ are finite unions of Rolle leaves of $d, d^{\alpha}$ and $d^{\beta}$ respectively.

Lemma 4 Suppose that $L$ is a connected component of $L^{\prime} \cap D$ and suppose that graph $\alpha$ is transverse to $d^{\prime}$. Then frL $\cap$ graph $\alpha$ is a clopen subset of $L^{\prime} \cap$ graph $\alpha$.

Proof Since $L^{\prime}$ is a Rolle leaf in $D^{\prime}$, it is closed in $D^{\prime}$ and so $L$ is closed in $D$. So, $\operatorname{fr} L \cap \operatorname{graph} \alpha=\operatorname{cl} L \cap \operatorname{graph} \alpha$ is closed in the graph of $\alpha$. Using the fact that $L^{\prime}$ is closed in $D^{\prime}$ again, we have cl $L \cap \operatorname{graph} \alpha \subseteq L^{\prime} \cap \operatorname{graph} \alpha$ and so $\operatorname{fr} L \cap \operatorname{graph} \alpha$ is a closed subset of $L^{\prime} \cap \operatorname{graph} \alpha$.

We now need to show that $\operatorname{fr} L \cap \operatorname{graph} \alpha$ is open in $L^{\prime} \cap \operatorname{graph} \alpha$, so let $p \in \operatorname{fr} L \cap \operatorname{graph} \alpha$. Let $L_{p}$ be the connected component of $L^{\prime} \cap$ graph $\alpha$ containing $p$. By the Frobenius theorem (see [8, Section 1]) there is a neighbourhood $U$ of $p$ and a diffeomorphism $\phi: \mathbb{R}^{n+1} \rightarrow U$ such that $\phi^{*} d^{\prime}=\operatorname{ker} d x_{n+1}$ and $\phi(0)=p$. Now, $L^{\prime}$ is a leaf of $d^{\prime}$ and $p \in L^{\prime} \cap U$, so the hyperplane $\mathbb{R}^{n} \times\{0\}$ is a component of $\phi^{-1}\left(L^{\prime} \cap U\right)$. Since $L^{\prime} \cap$ graph $\alpha \cap U$ is a submanifold of $L^{\prime} \cap U$, we can find an open box $B$ centred at 0 
such that $N:=\phi^{-1}\left(L^{\prime} \cap\right.$ graph $\left.\alpha \cap U\right) \cap B$ is connected. Let $B_{0}=\left(\mathbb{R}^{n} \times\{0\}\right) \cap B$. Then $N$ is a closed codimension one submanifold of $B_{0}$ and so $B_{0} \backslash N$ has exactly two components, $B_{1}$ and $B_{2}$, say. Since $p \in \mathrm{cl} L$, at least one of $B_{1}$ or $B_{2}$ must be contained in $\phi^{-1}(L \cap U)$. Also, $N=\operatorname{fr}\left(B_{i}\right) \cap B_{0}$ for each $i$ and so $\phi(N)$ is contained in $\operatorname{fr} L \cap \operatorname{graph} \alpha$. But $\phi(N)$ is open in $L^{\prime} \cap$ graph $\alpha$, by our choice of $B$, and the lemma is proved.

The following proposition suffices to prove the theorem.

Proposition 5 Let $L \subseteq \mathbb{R}^{n}$ be a Rolle leaf over $\mathcal{P}^{\prime}(\mathcal{R})$. Then $L$ is definable in $\mathcal{P}^{\prime}(\mathcal{R})$.

Proof The proof is by induction on $n$. The $n=1$ case is trivial, so we assume that $n>1$ and that the proposition is true for Rolle leaves over $\mathcal{P}^{\prime}(\mathcal{R})$ contained in $\mathbb{R}^{m}$ with $m<n$. Thus if $C \subseteq \mathbb{R}^{n}$ is a $C^{2}$ cell of dimension less than $n$ and $V \subseteq C$ is a Rolle leaf of a definable codimension one distribution on $C$, then $V$ is definable.

Suppose that $L \subseteq \mathbb{R}^{n}$ is a Rolle leaf over $\mathcal{P}^{\prime}(\mathcal{R})$. Then $L$ is a closed embedded proper submanifold of $\mathbb{R}^{n}$, and so there are $p \in \mathbb{R}^{n} \backslash L$ and $r>0$ such that $B(p, 2 r) \cap L=\emptyset$, where $B(a, \varepsilon)$ is the open ball around $a$ of radius $\varepsilon$. Perhaps after translating and stretching, we may assume that $p=0$ and that $r=1$. Let $\phi: \mathbb{R}^{n} \backslash\{0\} \rightarrow \mathbb{R}^{n} \backslash\{0\}$ be the semialgebraic diffeomorphism $\phi(x)=\frac{x}{\|x\|^{2}}$. Then $\phi(L)$ is contained in $B(0,1 / 2)$ and $\mathrm{cl}(\phi(L)) \subseteq \phi(L) \cup\{0\}$. So, after replacing $L$ by $\phi(L)$, we may assume that $L$ is a Rolle leaf of a definable integrable $(n-1)$-distribution $d$ on $B^{\prime}(0,1):=B(0,1) \backslash\{0\}$, that $L \subseteq B(0,1 / 2)$ and that $\mathrm{cl} L \subseteq L \cup\{0\}$.

Let $\Pi_{n-1}$ be the projection onto the first $n-1$ coordinates. For each coordinate permutation $\sigma$ on $\mathbb{R}^{n}$, the set $B_{\sigma}=\left\{x \in B^{\prime}(0,1):\left.\Pi_{n-1}\right|_{\sigma^{*}\left(d\left(\sigma^{-1}(x)\right)\right)}\right.$ has rank $\left.n-1\right\}$ is open and together these sets cover $B^{\prime}(0,1)$. So it suffices to show that $L \cap B_{\sigma}$ is definable for each $\sigma$. Fix $\sigma$, which we may assume to be the identity. Let $\mathcal{C}$ be a $C^{2}$ cell decomposition of $B^{\prime}(0,1)$ compatible with $B_{\text {id }}, B^{\prime}(0,1 / 2)$ and $d$. We show that $C \cap L$ is definable for each cell $C \in \mathcal{C}$ such that $C \subseteq B_{\text {id }}$. If $C \in \mathcal{C}$ is not open then $L \cap C$ is definable, by Fact 2 and the inductive hypothesis. So, suppose that $C \in \mathcal{C}$ is open and that $C \subseteq B_{\text {id }}$. Let $N$ be a component of $L \cap C$. Since $N$ is a Rolle leaf of $\left.d\right|_{C}$ and $C$ is a cell, $N$ is the graph of a function $f: \Pi_{n-1}(N) \rightarrow \mathbb{R}$. Let $\alpha, \beta: \Pi_{n-1}(C) \rightarrow \mathbb{R}$ be the functions such that graph $\alpha$ and $\operatorname{graph} \beta$ are the two cells in $\mathcal{C}$ forming the 'bottom' and 'top' of $C$. Then the graph of $\alpha$ is compatible with $d$ and so it is either tangent to $d$ or transverse to $d$. Since graph $\alpha$ is connected, if it is tangent to $d$, then either graph $\alpha \subseteq L$ or $L \cap \operatorname{graph} \alpha=\emptyset$. If the graph of $\alpha$ is transverse to $d$ then by Fact 2 and the inductive hypothesis, $L \cap$ graph $\alpha$ is definable. By 
Lemma 4, $\operatorname{fr} N \cap \operatorname{graph} \alpha$ is a clopen subset of $L \cap \operatorname{graph} \alpha$ and so $\operatorname{fr} N \cap \operatorname{graph} \alpha$ is also definable. This all also holds with the graph of $\beta$ in place of the graph of $\alpha$. Since $N$ is bounded and the graph of a continuous function, $x \in \operatorname{fr} \Pi_{n-1}(N)$ if and only if there is a $y$ such that $(x, y) \in \operatorname{fr} N$. So the set $\operatorname{fr}_{n-1}(N) \cap \Pi_{n-1}(C)$ is definable. Let $\mathcal{D}$ be a cell decomposition of $\Pi_{n-1}(C)$ compatible with $\operatorname{fr}_{n-1}(N) \cap \Pi_{n-1}(C)$. Then for each $D \in \mathcal{D}$ we either have $D \subseteq \Pi_{n-1}(N)$ or $D \cap \Pi_{n-1}(N)=\emptyset$. For each non-open cell $D \in \mathcal{D}$ such that $D \subseteq \Pi_{n-1}(N)$, let $E_{D}=\left(\left.\alpha\right|_{D},\left.\beta\right|_{D}\right)_{D}$. Take a cell decomposition of $E_{D}$ compatible with $d$. Let $E^{\prime}$ be a cell in this decomposition such that graph $\left.f\right|_{D} \cap E^{\prime}$ is non-empty. Then by Fact 2, graph $\left.f\right|_{D} \cap E^{\prime}$ is either a finite union of Rolle leaves of the pullback of $d$ to $E^{\prime}$ and so definable by the inductive hypothesis, or is equal to $E^{\prime}$ (in the case that $E^{\prime}$ is tangent to $d$ ). So the graph of $\left.f\right|_{D}$ is definable. Finally, for each open cell $D \in \mathcal{D}$ such that $D \subseteq \Pi_{n-1}(N)$, the restriction of $f$ to $D$ is Pfaffian over $\mathcal{P}^{\prime}(\mathcal{R})$ and so is definable by Lemma 3. So $N$ is definable, as required.

\section{Acknowledgements}

The first author was supported in part by an EPSRC postdoctoral fellowship and by EPSRC grant EP/E050441/1, and the second author is supported by NSERC.

\section{References}

[1] J Bochnak, M Coste, M-F Roy, Real Algebraic Geometry, Ergebnisse der Mathematik und ihrer Grenzgebiete (Series 3) 36, Springer-Verlag, Berlin, 1998; translated from the 1987 French original, revised by the authors.

[2] L van den Dries, Tame Topology and O-minimal Structures, London Mathematical Society Lecture Note Series 248, Cambridge University Press, 1998.

[3] A G Khovanskiü, Fewnomials, Translations of Mathematical Monographs 88, American Mathematical Society, Providence, RI, 1991, translated from the Russian by Smilka Zdravkovska.

[4] J-M Lion, J-P Rolin, Volumes, feuilles de Rolle de feuilletages analytiques et théorème de Wilkie, Ann. Fac. Sci. Toulouse Math. (6) 7 (1998), 93-112.

[5] J-M Lion, P Speissegger, The theorem of the complement for nested sub-Pfaffian sets, Duke Math. J. 155 (2010), 35-90.

[6] R Moussu, C Roche, Théorie de Hovanskil et problème de Dulac, Invent. Math. 105 (1991), 431-441; doi: 10.1007/BF01232274. 
[7] P Speissegger, The Pfaffian closure of an o-minimal structure, J. Reine Angew. Math. 508 (1999), 189-211.

[8] P Speissegger, Pfaffian sets and o-minimality, to appear in: Lecture Notes on O-minimal Structures and Real Analytic Geometry (eds. C. Miller, J.-P. Rolin and P. Speissegger), Fields Institute Communications, Springer, 2012.

[9] A J Wilkie, A theorem of the complement and some new o-minimal structures, Selecta Math. (N.S.) 5 (1999), 397-421.

Centre for Interdisciplinary Computational and Dynamical Analysis, School of Mathematics, University of Manchester, Oxford Road, Manchester, M13 9PL, UK.

Department of Mathematics \& Statistics, McMaster University, 1280 Main Street West, Hamilton, Ontario L8S 4K1, Canada.

gareth.jones-3@manchester.ac.uk, speisseg@math.mcmaster.ca

Received: 17 January 2011 Revised: 25 January 2012 